\title{
UNA BREVE REFLEXIÓN SOBRE FINANCIAMIENTO DE LA INVESTIGACIÓN CIENTÍFICA, FORMACIÓN DE RECURSOS HUMANOS Y PLAN DE INNOVACIÓN Y DESARROLLO
}

$\mathrm{E}$ 1 constante desafío a que la ciencia está acostumbrada con la generación de nuevos paradigmas se ve en ciertas ocasiones remecido por políticas de financiamiento. En Chile estamos viviendo una de estas ocasiones, pues dada la buena situación económica del país y las políticas de desarrollo se han destinado recursos para incentivar la "innovación" y el desarrollo. Sin embargo, frente a este desafío hay opiniones encontradas. Es así como "Innovación" es de un sentido tan amplio que los recursos destinados a este rubro pueden tener variados caminos, donde el esfuerzo se puede diluir de tal modo que el efecto no sea percibido. Desarrollo es algo más concreto, sobre lo que se puede tener una postura mucho más definida. La pregunta es ¿cómo "el día a día" de la ciencia puede enfrentar este desafío? Es claro para todos que el cuello de botella que tiene el desarrollo de la ciencia en Chile radica en sus recursos humanos y la falta de financiamiento de muy buenos proyectos de investigación y de postdoctorado. Haré un breve análisis de cómo estas dos actividades se encuentran enlazadas y cómo cada una de ellas contribuye al desarrollo e innovación del quehacer científico.

Para aumentar los recursos humanos, desde hace ya varios años la comunidad científica en conjunto con las universidades asumieron esta responsabilidad a través de la formación de programas de postgrado. Esto se concretó con el apoyo de científicos, universidades y políticos por medio de un programa de financiamiento apoyado por las Naciones Unidas. Este programa de formación de científicos continuó su crecimiento gracias al apoyo decidido de la Fundación Andes y del gobierno a través de becas. Desafortunadamente, el crecimiento ha sido lento y si se mira hacia el futuro se observa que en cinco años cerca del $50 \%$ de los científicos actuales deberán ser reemplazados, si se jubilan a la edad de 65 años.

El crecimiento de la ciencia ha obligado a un mayor perfeccionamiento de científicos en todo el mundo a través de postdoctorados, pues cuando el concepto de grupos de investigación despareció y los grupos se transformaron en un investigador principal capaz de financiar su propia investigación, a través de proyectos que le permitían mantener un laboratorio, hubo la necesidad de contar con el apoyo de investigadores calificados. Estas posiciones habitualmente las ocupan investigadores que hacen su postdoctorado, y estudiantes de doctorado y estudiantes de pregrado que se encuentran en etapa de formación. Es el financiamiento de estos proyectos de investigación de excelencia lo que permite contribuir a la formación de nuevos científicos, pues despierta el interés de los estudiantes y contribuye a una buena formación de científicos jóvenes. En Chile se están recibiendo alrededor de 300 estudiantes de doctorado por año, cantidad absolutamente insuficiente si se piensa solo en el reemplazo de los que se van. ¿Cómo contribuir entonces al desarrollo? No parece muy difícil, ya que una fórmula probada es el aumento del número de proyectos de investigación, de becas de doctorado y el aumento en forma significativa del número de proyectos de postdoctorado. Estas actividades están ligadas y dependen unas de otras, puesto que al aumentar el número de proyectos de investigación se aumenta en forma proporcional el número de plazas para postdoctorados, con sus respectivos proyectos de investigación, y se amplifica la capacidad de formar doctores. Esta acción además permite que los egresados de programas de doctorado se inserten en la actividad de investigación con sus propios proyectos de postdoctorado. El aumento de la capacidad de investigación y la "innovación" en áreas como la biotecnología se incrementaría por sí sola, pues hay que recordar que varias iniciativas en este sentido han sido abordadas con mucho éxito por científicos jóvenes, egresados de nuestros programas de postgrado. La transferencia tecnológica viene agregada cuando se hace ciencia sin apellidos, y solo funciona de esta manera, puesto que de otra forma las tecnologías se hacen obsoletas en muy corto tiempo. 
Los científicos no debemos mirar con recelo el desafío empresa-investigación, pues siempre se necesitará de científicos, ya sea en las universidades, en las empresas u otro tipo de unidades donde se realice investigación, siempre que esta sea de excelencia. Por esto, para hacer una inversión equilibrada dentro de un plan conocido, que recoja la opinión de la comunidad científica que pertenece a las entidades antes mencionadas, es necesario que el gobierno considere la formación de recursos humanos y el apoyo directo a proyectos de investigación involucrados en esta tarea, para la elaboración de los planes de "innovación y desarrollo", de manera que los recursos puedan ser invertidos de manera eficiente para el país. Un reparto de dineros para dejar contentos a todos solo es una forma política de distribución, y obviamente demagógico e ineficiente. Por ejemplo, no hemos escuchado a ninguno de los candidatos a la Presidencia de la República mostrar una posición frente al tema de la ciencia en Chile. Concluyo que el porcentaje ínfimo de los recursos provenientes del "Royalty Minero" que se están destinando a las dos actividades claves antes mencionadas, recursos humanos y financiamiento de la investigación, y que reitero son esenciales para un verdadero desarrollo científico y tecnológico, apunta más bien a una política de reparto, pues no se nos ha solicitado nuestra opinión. Pienso que una forma seria de pensar en el futuro de la ciencia y la tecnología es sobre la base de una ley apropiada que asegure el financiamiento en términos del gasto anual que se requiere. De esta manera se podrá planificar a más largo plazo y establecer los parámetros y programas que regulen la calidad de sus logros y productos directamente relacionados con el desarrollo del país.
Espero no desmotivarlos con esta pequeña reflexión, sino más bien incentivarlos a seguir luchando por el desarrollo de la ciencia. Aprovecho esta oportunidad para darles la más cordial bienvenida a la XLVIII Reunión Anual de la Sociedad de Biología de Chile, a la XII Reunión Anual de la Sociedad de Ecología de Chile y al XXVII Congreso Chileno de Microbiología. En esta reunión también participan con Simposios las Sociedades afiliadas de Bioquímica y Biología Molecular de Chile y la de Farmacología de Chile. Esta reunión, producto del esfuerzo conjunto de las tres Sociedades y del Directorio, promete ser del más alto nivel académico por la calidad de los invitados nacionales y extranjeros y de los trabajos que se presentan, donde habrá una activa participación de los estudiantes. Desgraciadamente, nos enteramos tardíamente que este año no contaríamos con fondos para el concurso de becas de ayuda a estudiantes de doctorado, que ofrecía tradicionalmente CONICYT. Ante esta situación, hemos asumido la responsabilidad de financiar a los estudiantes con fondos de otras ayudas, pues llamamos a concurso en el momento de la inscripción de los trabajos. Por esto, deseo agradecer a los socios que con su cuota hacen posible la Reunión, al Dr. Eric Goles y al Dr. Roberto Hojman por su generosa ayuda, y a las empresas que participan. En especial al Dr. Jorge Allende, quien muy generosamente trasladó a la Reunión los Simposios que tenía programados en Santiago.

\section{OCTAVIO MONASTERIO}

Presidente

Sociedad de Biología de Chile 